SCIENTIFIC REPORT

\title{
Post-brachytherapy initial tumour regression rate correlates with metastatic spread in posterior uveal melanoma
}

\author{
I Kaiserman, I Anteby, I Chowers, E Z Blumenthal, I Kliers, J Pe'er
}

Br J Ophthalmol 2004;88:892-895. doi: 10.1136/bjo.2003.036285

\begin{abstract}
Aim: To confirm the prognostic value of post-brachytherapy initial tumour regression rate in posterior uveal melanoma. Methods: A prospective, comparative, observational cohort study was carried out on 147 eyes (147 patients) with choroidal melanoma (mean age 61 years) treated with Ru106 brachytherapy. Observation procedure: Patients were followed clinically and ultrasonically every 6.7 (SD 0.3) months (1001 examinations). On average each patient was examined 5.8 times (mean follow up 9.6 (3.7) years). The echographic parameters included tumour base size, height, internal reflectivity, regularity, vascularity, and extrascleral extension. The clinical follow up included ocular examination and periodic metastatic screening (liver function tests and liver imaging). Main outcome measures were risk of liver metastasis in correlation with the post-brachytherapy initial tumour regression rate.

Results: At brachytherapy the mean tumour height was $5.2 \mathrm{~mm}$ (range 2.2-11.8 mm). After brachytherapy 142 tumours $(96.6 \%)$ responded by a decrease in height. The initial height regression rate was $6.1 \%(0.8 \%)$ per month in patients who later developed metastasis $v 4.3 \%(0.4 \%)$ per month in those who did not. Tumours higher than $6 \mathrm{~mm}$, tumours with an internal reflectivity smaller than $50 \%$, and tumours with an initial rate of height regression larger than $0.7 \mathrm{~mm} / \mathrm{month}$ (10\% per month) had higher 5 year melanoma related mortality. Kaplan-Meier survival analysis and the multivariate Cox proportional hazards model showed a significant role for tumour height and initial tumour regression rate on patients' survival.

Conclusion: This study confirms that post-brachytherapy initial tumour regression rate has a prognostic value.
\end{abstract}

M elanoma of the uvea is the most common primary intraocular malignancy in adults. In medium sized tumours, eye preserving treatments such as brachytherapy ${ }^{1}$ and external proton beam irradiation, ${ }^{2}$ which aim to destroy the tumour by irradiation, ${ }^{3}$ have been shown to be as effective as enucleation with regard to the long term survival and risk of metastatic spread. ${ }^{4}$ It has been shown that post-irradiation, most tumours shrink without disappearing completely. ${ }^{5}$

Uveal melanomas metastasise relatively late: the 5, 10, and 15 year survival rates based on tumour related mortality are reported to be $72 \%, 59 \%$, and 53\%, respectively, ${ }^{7}$ compared to age matched controls. However, the survival after clinical diagnosis of hepatic metastasis is poor. ${ }^{8}$ Thus, it is important to define clinical factors predictive of metastatic spread. Such unfavourable prognostic parameters include a larger tumour diameter, ${ }^{10}$ a faster growing tumour, ${ }^{11}$ presence of extrascleral growth, tumour margin location anterior to the equator of the eye, ${ }^{12}$ ciliary body involvement, ${ }^{13}$ older age, ${ }^{10}$ male sex, ${ }^{10}$ tumour induced glaucoma, ${ }^{14}$ and specific microvascular patterns $^{1015}$ which can be assessed clinically either by confocal indocyanine green angiography ${ }^{16-18}$ or ultrasound backscatter parameters. ${ }^{19}$

We have previously shown that, following brachytherapy, larger tumours shrink significantly faster than smaller ones. ${ }^{20}$ Thus, since larger tumours have a worse prognosis, one might assume that a fast shrinking tumour might be an unfavourable prognostic factor. While some previous studies did not support this notion, ${ }^{5}$ others have shown that the percentage of change in tumour height at 12 months post-brachytherapy does have a prognostic value. ${ }^{21}$

In the present study we looked at the correlation between post-brachytherapy initial tumour regression rate and the 5 year melanoma related mortality in order to statistically confirm what is considered to be a "common knowledge" that the tumour's response to brachytherapy can be used as a prognostic parameter.

\section{PATIENTS AND METHODS}

In all, 147 consecutive patients (80 women) who were diagnosed with uveal malignant melanoma between 1988 and 1998 and were treated with Ru-106 brachytherapy (10 000 cGy to the apex not exceeding 100000 cGy to the base) were included in the study. We excluded from the study patients with iris or iridociliary melanomas and patients with metastatic disease at the time of diagnosis.

Patients were followed at 3 months post-brachytherapy and every 6 months thereafter. The follow up included ocular examination, metastatic screening (liver function tests and liver imaging (ultrasound or computed tomography)), and ocular echographic examination (standardised A-scans and B-scans; B-Scan-S, Biovision International, ClermontFerrand, France). ${ }^{22}$ Echographically, we measured the tumour's height, base diameter, internal reflectivity (mean spikes height over the thickness of the tumour when the probe is perpendicular to the tumour's surface), location (posterior or anterior to the equator), regularity, vascularity and extrascleral extension. Echography was performed by several examiners using the same standardised echography machine.

Demographics (age, sex, country of origin) and treatment variables (total radiation dose to the tumour's base and apex (cGy) and the radiation rate (cGy/hour)) were collected from the patients' charts. The vital status of all patients (or their date of death) was confirmed from the records of the Israel cancer registry and the Israel population registry. Dead patients with liver metastases documented by liver ultrasound and computed tomography examinations were considered to have died of their disease unless another cause of death was specifically documented.

For the temporal dynamics analysis (see fig 3), the normalised tumour height (expressed in percentage of the height at brachytherapy) and the tumour's internal reflectivity (expressed in percentage of maximal scleral reflectivity) 
were grouped into 3 month groups and the mean and standard deviation (SD) of each group were calculated. These data were fitted with polynomial, hyperbolic, logarithmic, and first and second order exponential equations using the least square fitting method (Sigmaplot 2001, SPSS Inc, Chicago, IL, USA). The best fitting equation (determined by goodness of fit analysis) was found to be a first order exponential equation.

The amount of height decrease at the first postoperatve examination ( 3 months after brachytherapy) was used to calculate the "initial height regression rate."

Survival rates were plotted using the product limit method of Kaplan and Meier. ${ }^{23}$ Survival rates for each prognostic parameter were compared using the log rank test. ${ }^{24}$ The proportional hazards model of $\operatorname{Cox}^{25}$ was used to adjust for the simultaneous effects of the various covariates (SPSS version 9 (SPSS Inc, Chicago, IL, USA)). The parameters included in the model were age, sex, radiation dose to tumour's base, largest basal tumour dimension, tumour height, internal reflectivity, location of tumour, regularity of tumour, vascularity of tumour, initial post-brachytherapy tumour height regression.

\section{RESULTS}

A total of 147 patients ( 147 eyes) with a mean age of 61 years (range 29-97 years) were included in the study; 57 had small tumours (2-4 $\mathrm{mm}$ tumour height), 66 medium tumours $(4-8 \mathrm{~mm})$, and 24 large tumours $(>8 \mathrm{~mm})$. The postbrachytherapy follow up included A-scan and B-scan ultrasonography and a clinical examination (including metastatic evaluation) 3 months after the surgery and every $6.6(0.3)$ (mean (SE) ) months thereafter (a total of 1001 examinations). Each patient was examined on average 5.6 times (range 3-17). Thirty three of the 147 patients died during the follow up period (mean follow up 9.6 (3.7) years), 31 of them of metastatic melanoma (documented by liver imaging).

At the time of brachytherapy, mean tumour height was $5.7 \mathrm{~mm}$ (range 2.2-11.8 mm). Following brachytherapy, 142 $(96.6 \%)$ tumours decreased in height with an average initial rate of $0.28(0.03) \mathrm{mm} / \mathrm{month}(4.5 \%(0.4 \%)$ of original height) (mean (SE), median: $0.19 \mathrm{~mm} /$ month, 3.9\%). We grouped the patients into those who did and those who did not develop metastasis by the end of the follow up period. The non-metastatic group had a mean tumour height at brachytherapy of $5.4(0.2) \mathrm{mm}$ and regressed initially by 0.24 $(0.03) \mathrm{mm} / \mathrm{month}(4.3 \%(0.4 \%))$, while the metastatic group had a mean tumour height of $6.4(0.4) \mathrm{mm}$ and regressed initially by $0.42(0.05) \mathrm{mm} / \mathrm{month}(6.1 \%(0.8 \%))$ (Student's $t$ test for the rate of regression, $\mathrm{p}=0.03$ ).

Figure 1 presents the 5 year melanoma related mortality rate as a function of pre-brachytherapy tumour height (fig 1A), reflectivity (fig 1B) and initial post-brachytherapy tumour regression rate (figs $1 \mathrm{C}, \mathrm{D}$ ). Tumours higher than $6 \mathrm{~mm}$ or tumours with an internal reflectivity less than $50 \%$ had the highest 5 year melanoma related mortality $\left(\chi^{2}\right.$ test, $\mathrm{p}=0.04$ and $\mathrm{p}=0.1$ respectively). Moreover, tumours with an initial rate of height regression more than $0.7 \mathrm{~mm} / \mathrm{month}$ (fig lC) or $10 \%$ per month (fig lD) had a significantly higher ( $\chi^{2}$ test, $\mathrm{p}=0.04$ and $\mathrm{p}=0.03$ respectively) melanoma related mortality.

Figure 2 shows the Kaplan-Meier survival analysis ${ }^{23}$ stratified by height (fig 2A), reflectivity (fig $2 \mathrm{~B}$ ) and initial post-brachytherapy regression rate (figs 2C, D). As previously reported, ${ }^{10}$ tumour height had a significant impact on survival (log rank test for equality of survival distributions, $\mathrm{p}=0.04$ ). While tumours with an initial internal reflectivity of less than $30 \%$ seem to do worse on survival curves, the effect of the internal reflectivity was not statistically significant ( $\log$ rank test, $\mathrm{p}=0.8$ ). Both the initial absolute
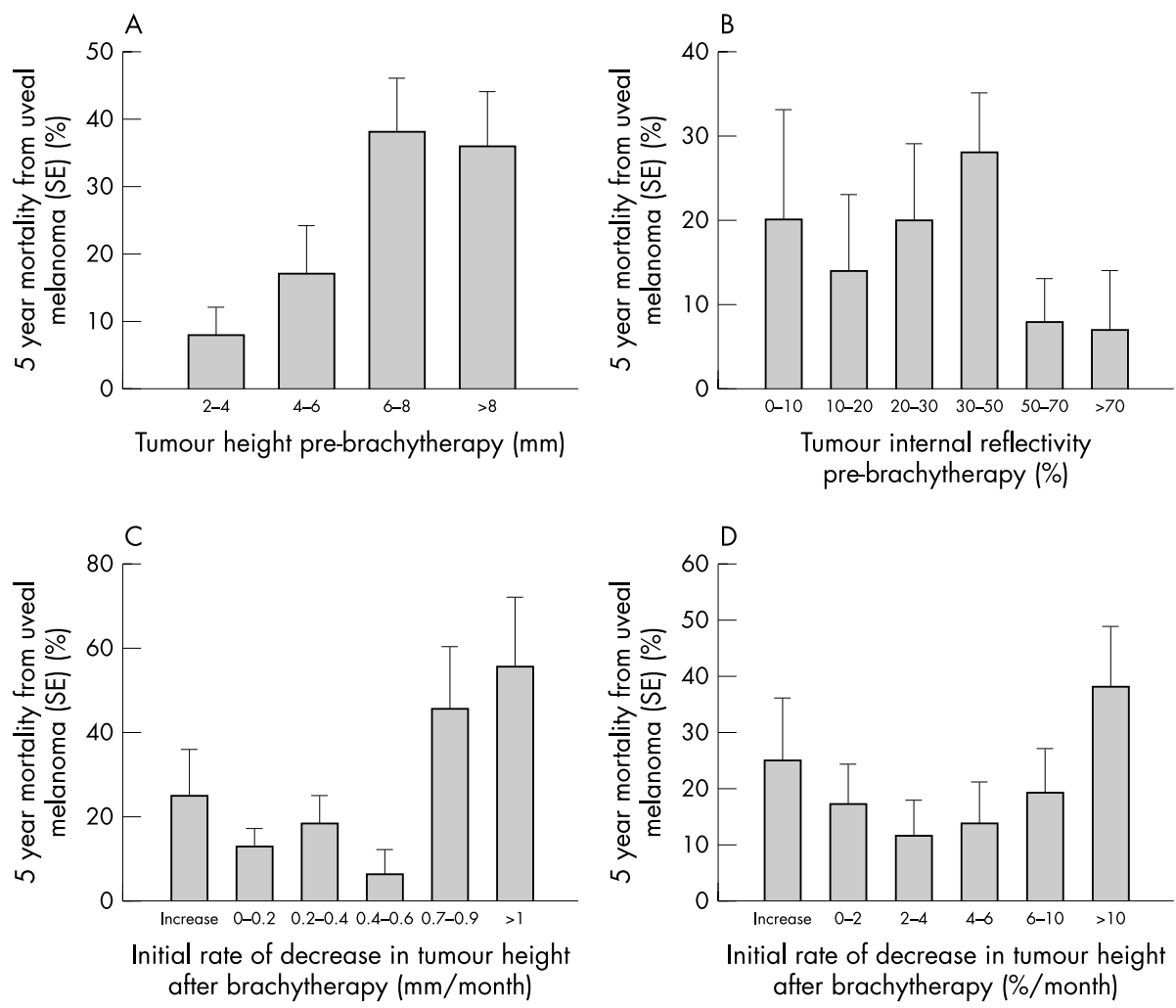

Figure 1 The 5 year rate mortality from uveal melanoma stratified by pre-brachytherapy tumour height (A), reflectivity (B), and initial postbrachytherapy rate of height decrease both in $\mathrm{mm}(C)$ and in percentage of the initial height (D). (A) $p=0.04,(B) p=0.1,(C) p=0.02,(D) p=0.04$. 

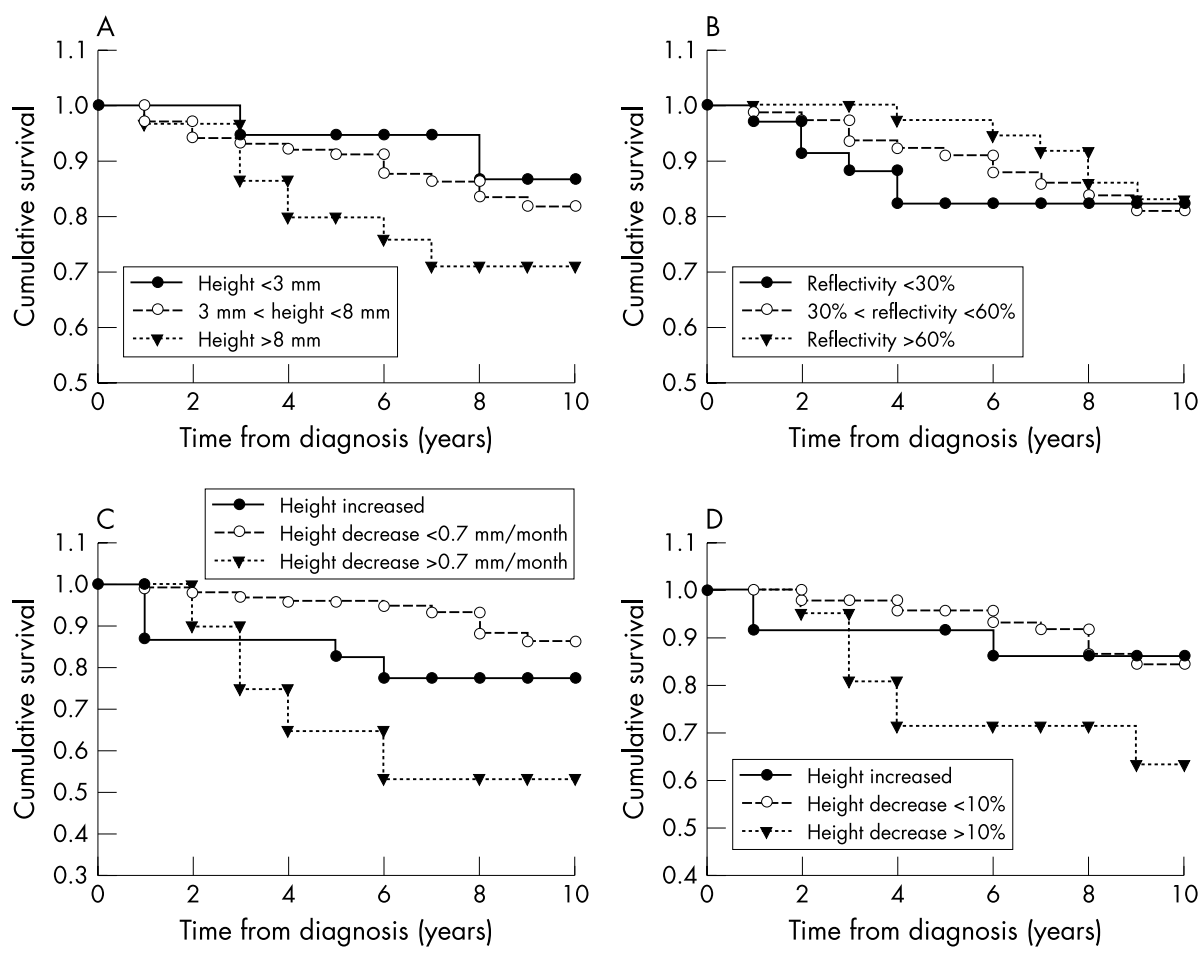

Figure 2 The Kaplan-Meier survival plot stratified by pre-brachytherapy tumour height (A), reflectivity (B), and initial post-brachytherapy rate of height decrease both in $\mathrm{mm}(C)$ and in percentage of the initial height (D). (A) $p=0.04,(B) p=0.8,(C) p=0.02,(D) p=0.04$.

regression rate (fig $2 \mathrm{C}$ ) and the initial proportional rate (fig 2D) had a significant impact on the 5 year melanoma related mortality (log rank test, $\mathrm{p}=0.02$ and $\mathrm{p}=0.04$ respectively).

Figure 3 shows the normalised post-brachytherapy tumour regression in the two groups of patients. The regression in the metastatic group could be fitted with the exponential equation: height $=53+46^{*} e^{-1.9 t}(t=$ time in years $)$, while the non-metastatic group was fitted with: height $=$ $71+27^{*} \mathrm{e}^{-2.1 t}$. Based on these equations, the metastatic group had a faster initial regression rate $(7.7 \% \vee 6.4 \%$ in the first month) and stabilised on a lower percentage of its initial height $(53 \% \vee 71 \%)$. The difference between the two groups was statistically significant (Student's $t$ test, $\mathrm{p}=0.01$ ). We compared the post-brachytherapy changes of internal reflectivity in the metastatic $v$ non-metastatic patients;

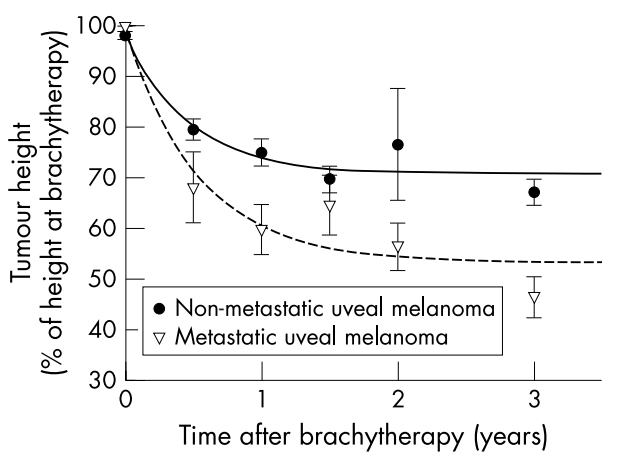

Figure 3 Post-brachytherapy tumour height dynamics of the nonmetastatic $v$ the metastatic patients. The solid line represents the function: height $=71+27^{*} \mathrm{e}^{-2.1 t}(\mathrm{t}$, time post-brachytherapy in years; height, percentage of tumour height at brachytherapy) and the broken line represents height $=53+46^{*} \mathrm{e}^{-1.9 t}$. The difference between the two groups is statistically significant $(p=0.01)$. however, no significant difference was noted between the two groups.

Multivariate Cox regression analysis indicated the most significant parameter to be tumour height (beta $=0.26$, $\mathrm{t}=8.8, \mathrm{p}=0.003)$ followed by the initial height regression rate $($ beta $=0.5, t=2.1 \mathrm{p}=0.04$ ). The other demographic and ultrasonic covariates did not reach the statistical significance limit.

\section{DISCUSSION}

Cruess et $a l^{5}$ have claimed that the rate of regression of the tumours in patients with metastatic melanoma was not appreciably different from the rate of regression in nonmetastatic patients, making it a poor indicator of prognosis. The same group subsequently found a prognostic value for the rate of regression of tumour thickness at 12 months posttreatment (but not earlier). ${ }^{21}$ Similar results were shown after proton beam irradiation. ${ }^{26}$ The present study suggests that the initial rate (during the first 3 months) of post-brachytherapy tumour regression has a prognostic value. Previous studies indicated a high prognostic value both for the tumour size ${ }^{10}$ and its growth rate. ${ }^{11}$ As larger, fast growing, tumours have a more intense proliferative activity, ${ }^{27}$ they are associated with a worse prognosis ${ }^{28}$ and were shown to be more radiosensitive. ${ }^{29}$ This might explain our findings that the more radiosensitive fast shrinking tumours had a worse prognosis.

Since larger tumours have both a worse prognosis ${ }^{10}$ and a faster post-brachytherapy regression rate, ${ }^{20}$ we used multivariate Cox regression analysis in order to evaluate the individual contribution of the regression rate on prognosis. We have shown that although height is the more significant prognostic variable, the initial regression rate had an additional, albeit small, independent value in prognosis. As the radiation dose to the base was also included in the Cox model we might conclude that the prognostic value of the initial tumour regression rate does not result from different radiation doses as was suggested previously. ${ }^{30}$ 
In conclusion, as in recent years new treatment avenues are being explored for metastatic uveal melanoma, ${ }^{31}{ }^{32}$ the initial post-brachytherapy tumour's regression rate can be useful in estimating the hazard of metastatic spread, deciding on the follow up regimen, and possibly employing preventive treatment in the future.

\section{Authors' affiliations}

I Kaiserman, I Anteby, I Chowers, E Z Blumenthal, I Kliers, J Pe'er, Department of Ophthalmology, Hadassah University Hospital, Jerusalem, Israel

Correspondence to: Dr I Kaiserman, Department of Ophthalmology, Hadassah University Hospital, POB 12000, IL-91120 Jerusalem, Israel; lgork@cc.huji.ac.il

Accepted for publication 11 December 2003

\section{REFERENCES}

1 Packer S, Rotman M. Radiotherapy of choroidal melanoma with iodine 125 Int Ophthalmol Clin 1980;20:135-42.

2 Gragoudas ES, Goitein M, Verhey L, et al. Proton beam irradiation. An alternative to enucleation for intraocular melanomas. Ophthalmology 1980;87:571-81.

3 Rotman M, Long RS, Chan B, et al. Radiation therapy of choroidal melanoma. In: Hilaris BS, ed. Afterloading 1955;IN922RH-1975a:133-9.

4 Diener West M, Earle JD, Fine SL, et al. The COMS randomized trial of iodine 125 brachytherapy for choroidal melanoma, III: initial mortality findings. COMS Report No 18. Arch Ophthalmol 2001;119:969-82.

5 Cruess AF, Augsburger JJ, Shields JA, et al. Regression of posterior uveal melanomas following cobalt-60 plaque radiotherapy. Ophthalmology 1984;91:1716-9.

6 Diener West M, Hawkins BS, Markowitz JA, et al. A review of mortality from choroidal melanoma. II. A meta-analysis of 5-year mortality rates following enucleation, 1966 through 1988. Arch Ophthalmol 1992;1 10:245-50.

7 Gamel JW, McLean IW, McCurdy JB. Biologic distinctions between cure and time to death in 2892 patients with intraocular melanoma. Cancer 1993;71:2299-305.

8 Seddon JM, Albert DM, Lavin PT, et al. A prognostic factor study of diseasefree interval and survival following enucleation for uveal melanoma. Arch Ophthalmol 1983;101:1894-9.

9 Kath R, Hayungs J, Bornfeld N, et al. Prognosis and treatment of disseminated uveal melanoma [see comments]. Cancer 1993;72:2219-23.

10 Folberg R, Pe'er J, Gruman LM, et al. The morphologic characteristics of tumor blood vessels as a marker of tumor progression in primary human uveal melanoma: a matched case-control study. Hum Pathol 1992;23:1298-305.

11 Char DH, Kroll S, Phillips TL. Uveal melanoma. Growth rate and prognosis. Arch Ophthalmol 1997;115:1014-18.

12 McLean IW, Foster WD, Zimmerman LE. Uveal melanoma: location, size, cell type, and enucleation as risk factors in metastasis. Hum Pathol 1982;13:123-32.
13 Li W, Gragoudas ES, Egan KM. Metastatic melanoma death rates by anatomic site after proton beam irradiation for uveal melanoma. Arch Ophthalmol 2000; 1 18:1066-70.

14 Coleman K, Baak JP, Van Diest P, et al. Prognostic factors following enucleation of 111 uveal melanomas [see comments]. Br J Ophthalmol 1993;77:688-92

15 Folberg R, Rummelt $V$, Parys Van Ginderdeuren $R$, et al. The prognostic value of tumor blood vessel morphology in primary uveal melanoma. Ophthalmology 1993;100:1389-98.

16 Mueller AJ, Freeman WR, Schaller UC, et al. Complex microcirculation patterns detected by confocal indocyanine green angiography predict time to growth of small choroidal melanocytic tumors: MuSIC Report II. Ophthalmology 2002;109:2207-14.

17 Mueller AJ, Bartsch DU, Folberg R, et al. Imaging the microvasculature of choroidal melanomas with confocal indocyanine green scanning laser ophthalmoscopy. Arch Ophthalmol 1998;1 16:31-9.

18 Mueller AJ, Bartsch DU, Schaller U, et al. Imaging the microcirculation of untreated and treated human choroidal melanomas. Int Ophthalmol 2001;23:385-93

19 Silverman RH, Folberg R, Boldt HC, et al. Correlation of ultrasound parameter imaging with microcirculatory patterns in uveal melanomas. Ultrasound Med Biol 1997; 23:573-81.

20 Kaiserman I, Anteby I, Chowers I, et al. Changes in ultrasound findings in posterior uveal melanoma after Ruthenium 106 brachytherapy. Ophthalmology 2002;109:1137-41.

21 Augsburger JJ, Gamel JW, Shields JA, et al. Post-irradiation regression of choroidal melanomas as a risk factor for death from metastatic disease. Ophthalmology 1987;94:1173-7.

22 Ossoinig KC. Standardized echography: basic principles, clinical applications, and results. Int Ophthalmol Clin 1979;19:127-210.

23 Kaplan EL, Meier P. Nonparametric estimation from incomplete observations. J Am Stat Assoc 1958:53:457-81.

24 Mantel N. Evaluation of survival data and two new rank order statistics arising in its consideration. Cancer Chemother Rep 1966;50:163-70.

25 Cox DR. Regression models and life-tables. J R Stat Soc B 1972;34:187-220.

26 Glynn RJ, Seddon JM, Gragoudas ES, et al. Evaluation of tumor regression and other prognostic factors for early and late metastasis after proton irradiation of uveal melanoma. Ophthalmology 1989;96:1566-73.

27 Chiquet C, Grange JD, Ayzac L, et al. Effects of proton beam irradiation on uveal melanomas: a comparative study of $\mathrm{Ki}-67$ expression in irradiated versus non-irradiated melanomas. Br J Ophthalmol 2000;84:98-102.

28 Seregard S, Oskarsson M, Spangberg B. PC-10 as a predictor of prognosis after antigen retrieval in posterior uveal melanoma. Invest Ophthalmol Vis Sci 1996;37:1451-8.

29 Grange JD, Thacoor S, Bievelez B, et al. [Comparative study of the tumor regression rate of 127 uveal melanomas irradiated with $106 \mathrm{Ru} / 106 \mathrm{Rh}$. Attempted analysis of the correlations between per-operative cytology and histopathology of the enucleated eye and the tumoral regression on the one hand, and general prognosis on the other hand]. Ophtalmologie 1990;4:221-4.

30 Kindy Degnan NA, Char DH, Castro JR, et al. Effect of various doses of radiation for uveal melanoma on regression, visual acuity, complications, and survival. Am J Ophthalmol 1989;107:114-20.

31 Aoyama T, Mastrangelo MJ, Berd D, et al. Protracted survival after resection of metastatic uveal melanoma. Cancer 2000;89:1561-8.

32 Woll E, Bedikian A, Legha SS. Uveal melanoma: natural history and treatment options for metastatic disease. Melanoma Res 1999;9:575-81. 